\title{
Time- and space-resolved spectroscopy of dynamic hohlraum interiors
}

\author{
J.E. Bailey ${ }^{\text {a,* }}$, G.A. Chandler ${ }^{\text {a }}$, G.A. Rochau ${ }^{\text {a }}$, Y. Maron ${ }^{\text {b }}$, S.A. Slutz ${ }^{\text {a }}$, G.S. Dunham ${ }^{\text {c }}$, \\ I. Golovkin ${ }^{\mathrm{d}}$, P.W. Lake ${ }^{\mathrm{a}}$, R.W. Lemke ${ }^{\mathrm{a}}$, J.M. Lucas ${ }^{\mathrm{c}}$, J.J. MacFarlane ${ }^{\mathrm{d}}$, T.A. Mehlhorn ${ }^{\text {a }}$, \\ T.C. Moore ${ }^{\text {c }}$, D.G. Schroen ${ }^{\text {e }}$, E. Stambulchik ${ }^{\text {b }}$, K. Youngblood ${ }^{\mathrm{e}}$ \\ a Sandia National Laboratories, Albuquerque, NM 87185-1196, USA \\ ${ }^{\mathrm{b}}$ Weizmann Institute of Science, Rehovot, Israel \\ ${ }^{\mathrm{c}}$ K-Tech Corporation, Albuquerque, NM 87185, USA \\ ${ }^{\mathrm{d}}$ Prism Computational Science, Madison, WI 53703, USA \\ ${ }^{\mathrm{e}}$ Schafer Corporation, Livermore, CA 94550, USA
}

Received 28 July 2005

Available online 30 September 2005

\begin{abstract}
A dynamic hohlraum is created when an annular z-pinch plasma implodes onto a cylindrical $0.014 \mathrm{~g} / \mathrm{cc}$ 6-mm-diameter $\mathrm{CH}_{2}$ foam. The impact launches a radiating shock that propagates toward the axis at $\sim 350 \mu \mathrm{m} / \mathrm{ns}$. The radiation trapped by the tungsten $z$-pinch plasma forms a $\sim 200 \mathrm{eV}$ hohlraum that provides X-rays for indirect drive inertial confinement fusion capsule implosion experiments. We are developing the ability to diagnose the hohlraum interior using emission and absorption spectroscopy of Si atoms added as a tracer to the central portion of the foam. Time- and space-resolved Si spectra are recorded with an elliptical crystal spectrometer viewing the cylindrical hohlraum endon. A rectangular aperture at the end of the hohlraum restricts the field of view so that the 1D spectrometer resolution corresponds approximately to the hohlraum radial direction. This enables distinguishing between spectra from the unshocked radiation-heated foam and from the shocked foam. Typical spectral lines observed include the Si Ly $\alpha$ with its He-like satellites and the He-like resonance sequence including He $\alpha$, He $\beta$, and $\mathrm{He} \gamma$, along with some of their associated Li-like satellites. Work is in progress to infer the hohlraum conditions using collisional-radiative modeling that accounts for the radiation environment and includes both opacity effects and detailed Stark broadening calculations. These 6-mm-scale radiation-heated plasmas might eventually also prove suitable for testing Stark broadening line profile calculations or for opacity measurements. (C) 2005 Published by Elsevier B.V.
\end{abstract}

Keywords: Inertial confinement fusion; K-shell spectra; Hohlraum

\section{Introduction}

A dynamic hohlraum is formed when a cylindrical annular $z$-pinch plasma is accelerated onto a low density cylindrical $\mathrm{CH}_{2}$ foam located at the axis [1-3]. The pinch plasma impact launches a radiating shock in the $\mathrm{CH}_{2}$ foam that propagates toward the axis at $\sim 350 \mu \mathrm{m} / \mathrm{ns}$. The $z$-pinch is composed of a high- $\mathrm{Z}$ element such as tungsten. The radiation is trapped by the tungsten plasma, forming a hohlraum (Fig. 1). Inertial confinement fusion (ICF) capsule implosions can be driven by the radiation that exists at the hohlraum center, prior to

\footnotetext{
* Corresponding author. Tel.: +1 5058457203 .

E-mail address: jebaile@sandia.gov (J.E. Bailey).
}

the arrival of the radiating shock. Such implosions are an interesting ICF approach because the energy delivered to the capsule can be large. Recent experiments have imploded capsules that absorbed more than $40 \mathrm{~kJ}$ of $\mathrm{X}$-rays, which is within an order of magnitude of the energy believed necessary for ICF ignition [4-6]. However, successful ICF requires precise control over the radiation symmetry, drive pulse time history, and peak radiation temperatures exceeding $250 \mathrm{eV}$, in addition to large capsule absorbed energies. The eventual development of methods to address these needs depends on accurate diagnosis, understanding, and control over the complex physics that exists within the dynamic hohlraum interior.

In this paper we describe the progress toward development of $\mathrm{Si}$ tracer spectroscopy as a diagnostic of the dynamic 


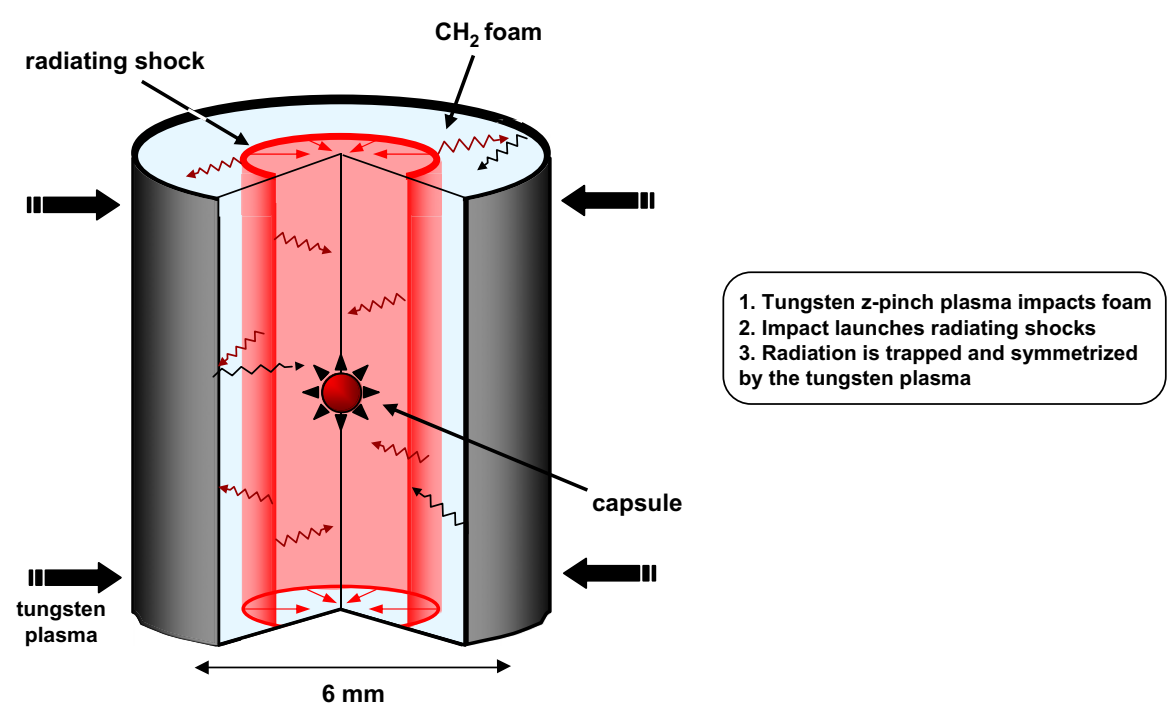

Fig. 1. Schematic diagram of a dynamic hohlraum ICF experiment.

hohlraum conditions. The basic idea is to add trace amounts of an element that will provide K-shell emission or absorption lines, record spectra using a time- and space-resolved spectrometer, and then use an atomic spectral model to infer the hohlraum conditions. This method offers advantages such as the ability to diagnose local conditions ahead and behind the shock, to provide detailed information about the shock characteristics, and the possibility of inferring the radiation heating that exists at the center of the hohlraum where an ICF capsule would be located. In addition, such data can be used to test understanding by comparing measured spectra with post-processed simulations. The challenges that must be addressed in order to achieve these benefits are numerous. First, targets must be constructed and experiments designed that enable the acquisition of high-quality space- and time-resolved tracer spectra. Once this is accomplished, analysis accounting for the collisional and radiative line excitation mechanisms, line broadening, and line transport must be performed. The radiative processes affecting the level populations require careful consideration, since excitations may occur both because of the quasi-Planckian thermal radiation trapped within the hohlraum and excitations by non-Planckian radiation due, for example, to strong line emission. The possible importance of non-equilibrium effects, such as rapid recombination that might occur behind the shock, must eventually be evaluated.

Cylindrical $14 \mathrm{mg} / \mathrm{cc}$ foam targets were constructed with an $\mathrm{SiO}_{1.5}$ tracer added at $\sim 1 \%$ atomic level. Experiments were conducted first with $\mathrm{SiO}_{1.5}$ dopant uniformly distributed throughout the foam and subsequently with the dopant confined to a 6-mm-tall region in the middle of the cylinder. Time- and space-resolved spectra were recorded using an elliptical crystal spectrometer equipped with an array of six imaging slits that project spectrally resolved images onto six time-gated microchannel plate (MCP) strip lines. An analysis strategy that employs a combination of calculations with the PRISMSPECT spectral analysis code and a code developed at the Weizmann Institute of Science (WIS) is under development. Calculations with the WIS model indicate that the spectra emerging from the region ahead of the shock are strongly influenced by photoexcitation and photoionization driven by $\mathrm{Si}$ line photons emitted from behind the shock. The determination of the plasma parameters from the spectra is not yet complete, but the tracer spectroscopy approach appears promising.

\section{Dynamic hohlraum simulations}

The experiments were designed using computer simulations $[4,7]$ as a guide for the plasma conditions that exist within the dynamic hohlraum. The simulations were performed using the $2 \mathrm{D}$ radiation-hydrodynamics code LASNEX [7,8]. They assume azimuthal symmetry and resolve the $R-Z$ plane with a Lagrangian mesh. Radiation is treated using a multi-group flux-limited diffusion model that assumes Local Thermodynamic Equilibrium (LTE). These simulations used an accurate representation of the experiment configuration described below, but the foam was assumed to be $\mathrm{CH}_{2}$ with no $\mathrm{SiO}_{1.5}$ dopant. It is very much possible that the addition of the dopant modifies the properties of the dynamic hohlraum plasma to some extent. For example, doped foam probably radiates more efficiently and the temperature behind the shock front may consequently be lower. Therefore, these simulations are used to guide expectations of the conditions that exist, not as definitive predictions for the conditions. The simulations are post-processed to create synthetic images of the emission emerging from the top of the dynamic hohlraum. At a given time the synthetic images are dominated by circular emission that arises from the shocked foam. The simulation timing is determined by measuring the radius of the circular shock emission, plotting the radius as a function of time, and comparing it to the radius inferred from time-resolved pinhole camera measurements (see Refs. [9,7] for further details). In the experiments described here we define time $=0$ to be the moment when the X-ray power, as measured by an XRD, emitted to the side of the $z$-pinch reaches its peak. Note that 
with this time definition, typical capsule implosions are completed at approximately $t=-4 \mathrm{~ns}$ to $-3 \mathrm{~ns}$ [5].

The simulation results for the foam density and temperature spatial profiles at the moment just prior to the arrival of the tungsten plasma at the initial foam radius are shown in Fig. 2. During the 95-ns-duration radially-inward z-pinch implosion, the tungsten plasma emits enough radiation to cause ablation of the foam. This ablation launches a weak shock into the foam, prior to the main shock launched by the impact of the tungsten. The characteristics of the initial ablation shock can be important, since the ablation implies that the main shock forms in plasma that is altered from its original conditions.

The simulated evolution of the foam density and temperature as the main shock forms is shown in Fig. 3. According to the simulations, the electron temperature just behind the main shock is initially $(t=-8.1 \mathrm{~ns})$ of order $800 \mathrm{eV}$. The plasma within the dynamic hohlraum interior is optically thin for the 100-1000 eV continuum photons that transport most of the energy and the radiation temperature is approximately constant with radius. The electron temperature is approximately the same as the radiation temperature for the region ahead of the shock. However, the predicted high electron temperature behind the shock implies that the shocked plasma is far from equilibrium with the radiation. Therefore, adding a mid- $Z$ or high- $Z$ dopant to increase the radiation efficiency could conceivably help increase the total radiation energy available in the hohlraum. As the shock moves inward, the electron temperature behind the shock is predicted to gradually decrease. The radiation temperature within the hohlraum simultaneously rises, reaching $\sim 240 \mathrm{eV}$ at $t=-4.1 \mathrm{~ns}$. The simulated electron density of the shocked foam increases to approximately $n_{\mathrm{e}} \sim 5.5 \times 10^{22} \mathrm{~cm}^{-3}$ at $t=-4.1 \mathrm{~ns}$, about
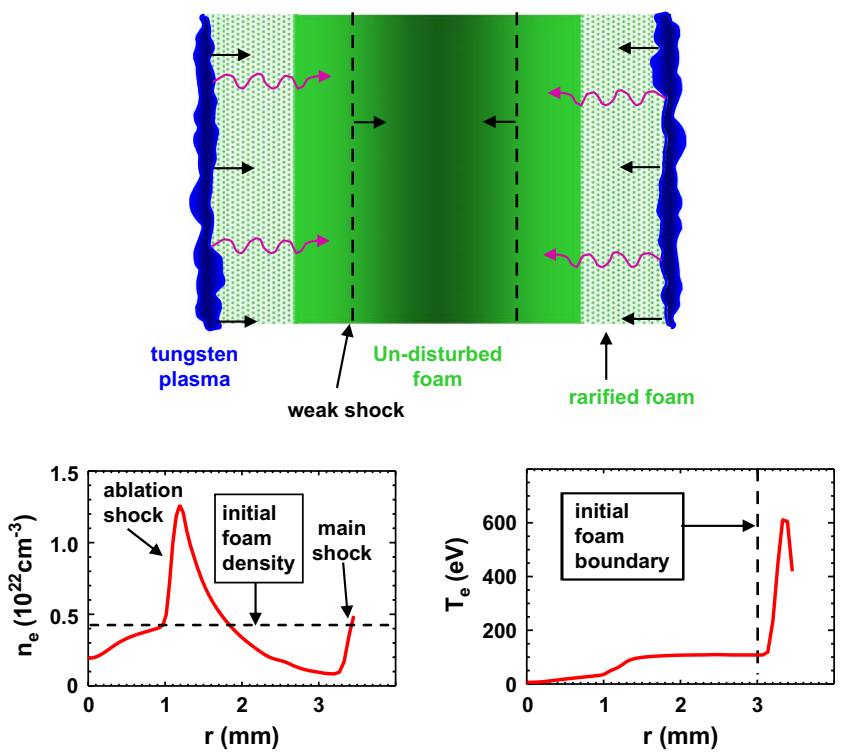

Fig. 2. Conceptual diagram and simulation predictions of dynamic hohlraum conditions just prior to tungsten plasma impact onto the central foam. The simulations correspond to pure $\mathrm{CH}_{2}$ foam, not the doped foam used in the experiments described here. A weak shock has been launched in the foam due to ablation by $z$-pinch plasma run-in radiation.
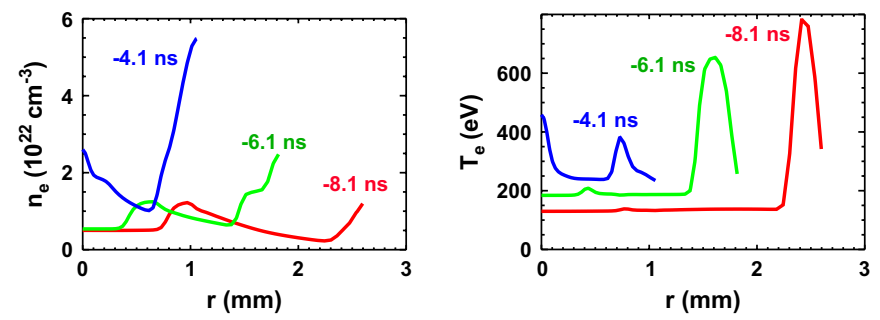

Fig. 3. Simulation predictions of the evolution in dynamic hohlraum conditions after the main shock is launched in the foam. According to the simulations, the main shock is initially created in rarified plasma, due to the initial weak early ablation shock. The radiation temperature ahead of the shock is approximately equal to the electron temperature. The times refer to experiment Z1291 (see Fig. 7).

an order of magnitude higher than the initial density of the unperturbed foam. Note that according to the simulations, the weak early ablation shock reaches the axis at $t=-4.1 \mathrm{~ns}$, approximately the time when capsule implosions are beginning to reach maximum compression. According to $2 \mathrm{D}$ integrated simulations described elsewhere [10], this early ablation driven shock is too weak to perturb the implosion, but clearly this is an issue that would be valuable to evaluate experimentally.

Examination of these simulations helps define the questions that tracer spectroscopy measurements should attempt to answer. These are as follows: is there an early ablation driven shock, and if so, what are its characteristics? What are $n_{\mathrm{e}}$ and $T_{\mathrm{e}}$ behind the shock front? What are $n_{\mathrm{e}}$ and $T_{\mathrm{e}}$ ahead of the main shock? What is the radiation temperature $T_{\mathrm{r}}$ throughout the hohlraum? Is the hohlraum uniform? How fast does the shock travel? How rapidly does the material behind the shock cool? How much separation exists between the shock and the tungsten plasma hohlraum wall? Answering these questions experimentally is a formidable challenge, yet it is probably a requirement for optimizing the drive available for ICF capsule implosions.

\section{Experiment configuration}

The experiments used the $\mathrm{Z}$ accelerator [1] to drive a dynamic hohlraum configuration similar to Bailey et al. [5]. It consisted of nested concentric cylindrical tungsten wire arrays with 240 wires in the 20 -mm-radius outer array and 120 wires in the 10-mm-radius inner array. The wire diameter was $7.5 \mu \mathrm{m}$. A $12-\mathrm{mm}$-tall $6-\mathrm{mm}$-diameter $14 \mathrm{mg} / \mathrm{cc}$ foam cylinder was positioned at the $z$-pinch axis. The bulk of the foam was $\mathrm{CH}_{2}$, with $\sim 1 \%$ by atom $\mathrm{SiO}_{1.5}$ tracer added. A new technique was developed that enabled adding the tracer on an atomic basis, rather than adding $\mathrm{SiO}_{1.5}$ particles to a $\mathrm{CH}_{2}$ foam. This was accomplished by mixing an organometallic $\mathrm{SiO}_{1.5}$ solution to the normal $\mathrm{CH}_{2}$ solution used to cast the foam. This method can produce foams that retain the $14 \mathrm{mg} /$ cc density normally used in our pure $\mathrm{CH}_{2}$ foam experiments. This method provides a foundation for future experiments using other higher- $Z$ dopants, since many other compounds are available in organometallic form. 
The primary diagnostic used in these experiments was a time- and space-resolved elliptical crystal spectrometer viewing the top of the cylindrical foam target, along the $z$-pinch axis [11,12]. An array of six 50- $\mu$ m-wide spaceresolving slits projected magnification $=0.5$ spectrally resolved images onto six time-gated MCP strip lines. The spatial resolution was approximately $\sim 220 \mu \mathrm{m}$ and the field of view was $\sim 8 \mathrm{~mm}$ at the source. The spectral dispersion was provided by a $3 \mathrm{~cm} \times 10 \mathrm{~cm}$ Pentaerythritol (PET) crystal bent to an ellipse with eccentricity $=0.976$ and a $288.0 \mathrm{~cm}$ focal length. The spectral range covered was approximately $5.1-6.7 \AA$ and the resolving power was approximately $\lambda / \delta \lambda \sim 800$. The time gate was generated by a $\sim 1$ ns FWHM, $\sim 400 \mathrm{~V}$ pulse in initial experiments and a $\sim 300$ ps FWHM, $700 \mathrm{~V}$ pulse in subsequent experiments. In both cases the voltage was applied to the 4-mm-wide strip lines in an open circuit configuration. The time resolution was estimated to be $\sim 0.5 \mathrm{~ns}$ with a $1 \mathrm{~ns}$ or 2 ns interval between frames. The spectra were recorded on Kodak TMAX film and the film response was unfolded using a calibrated step wedge. The spectra were processed by first creating a separate image for each frame. The data were corrected for the space direction deviations caused by crystal imperfections [12] and the wavelength scale was applied using the instrument geometry with the $\operatorname{Ly} \alpha(\lambda=6.180 \AA)$ as a reference. The spectral intensity is corrected for the filter transmission, the crystal reflectivity, and the instrument geometry. No correction was applied for the MCP photocathode efficiency as calibrations of the actual response were unavailable. This could lead to errors when comparing spectral lines separated by $\sim 1 \AA$, but should be relatively unimportant for closely spaced lines.

A variety of other diagnostics were employed as secondary instruments [13]. The self-emission from the shock was measured with a time-gated filtered $\mathrm{X}$-ray pinhole camera viewing along the target axis from below. Arrays of filtered X-ray diodes (XRDs) and filtered diamond photoconducting detectors measured the X-ray emission from the top, bottom and side.
Two space-resolving time integrated convex crystal spectrometers viewed the $\mathrm{Si}$ emission from the top of the cylinder.

\section{Results}

Initial proof-of-principle experiments used a dopant that was uniformly distributed throughout the foam. Data from a uniform dopant experiment (Z1117) confirmed that such foams produced measurable Si line emission (Figs. 4 and 5). The first four frames at $-6.7 \mathrm{~ns}$ to $-3.7 \mathrm{~ns}$ span the period leading to up peak compression of a typical ICF implosion experiment. The shock velocity was the same as in previous experiments, but in all the experiments presented here the shock timing relative to the side on XRD peak was earlier than in previous experiments by approximately $1.5 \mathrm{~ns}$. This timing difference may be because the side on XRD peak is partially controlled by random aspects of the z-pinch stagnation physics that are unrelated to the dynamic hohlraum shock trajectory. It is presently unknown whether this difference might also have a contribution from a difference in the sample, e.g., doping the foam with $\mathrm{SiO}_{1.5}$, or from a timing error in one or the other set of experiments. The Ly $\alpha$ is the brightest feature observed in the data shown in Figs. 4 and 5. He $\beta, \operatorname{He} \gamma$, and He $\delta$ were also observed in emission. The latter transitions consist of a broad emission peak with a self-absorption dip. A likely cause of the broadening is opacity; the line center optical depths calculated using the conditions expected from the simulations are up to $\sim 1000$. The origin of the self-absorption dip in the He-like features is possibly due to cooling plasma that jets out of the diagnostic aperture. The decrease in Ly $\alpha$ signal intensity with time may be consistent with the simulation results suggesting that the $T_{\mathrm{e}}$ decreases with time. However, this signal is also affected by the chordal integration of the $1 \mathrm{D}$ imaging spectrometer viewing a circular object with a radius that decreases with time. In addition, the variation of MCP detector sensitivity must be evaluated before any strong conclusions are drawn.

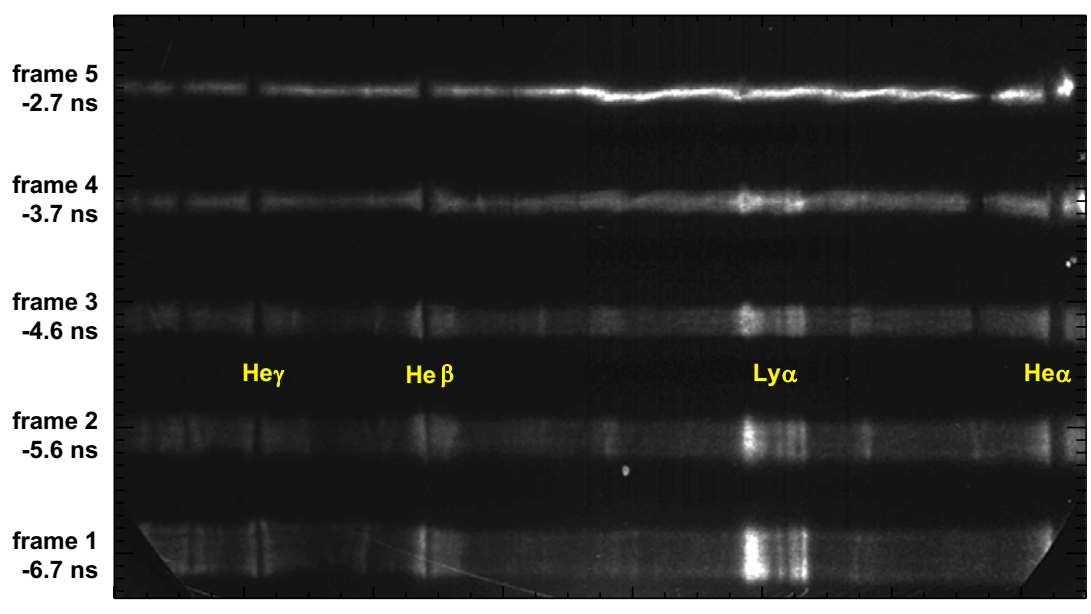

$\lambda$

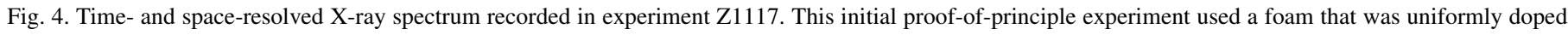

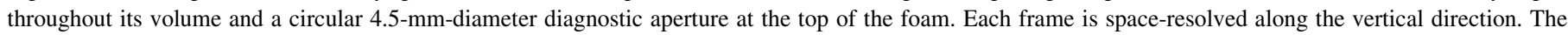
wavelength axis is horizontal. The times are measured with respect to the peak in the side on X-ray power. 


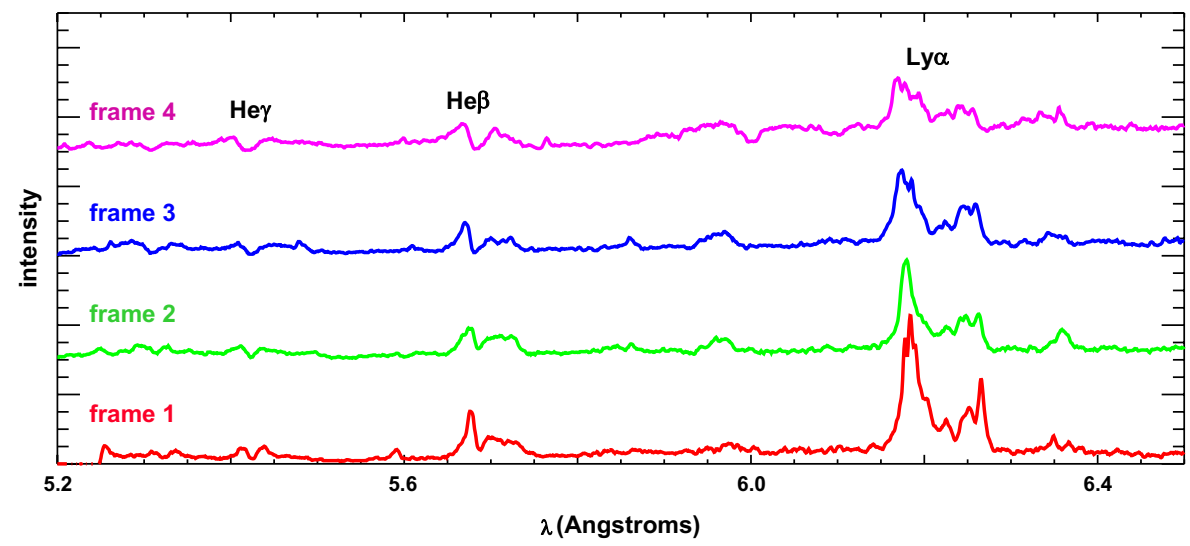

Fig. 5. Lineouts of the uniform dopant Z1117 spectra. Each lineout averages over a 0.6-mm-tall region at the top of the spectral image. This region corresponds approximately to the emission from the shocked foam.

The initial experiments, e.g., as shown in Figs. 4 and 5, were performed using a circular 4.5-mm-diameter diagnostic aperture at the top of the foam. This maximizes the signal, but it implies that each spatial resolution element of the spectra is composed of contributions from a variety of radii, since a 1D resolving instrument integrates over chords parallel to the dispersion plane. This makes it difficult to differentiate between emission arising from the shocked plasma and the unshocked plasma. In subsequent experiments a rectangular aperture was used to alleviate this problem, as illustrated in Fig. 6. The rectangular aperture restricts the field of view so that the spatial resolution corresponds more closely to the radial coordinate of the cylinder. This approximation remains valid as long as the radius of interest is large compared to the half width of the rectangular aperture. This enables unambiguous discrimination between the emission from shocked and unshocked foam, a critical capability if we are to

(a)

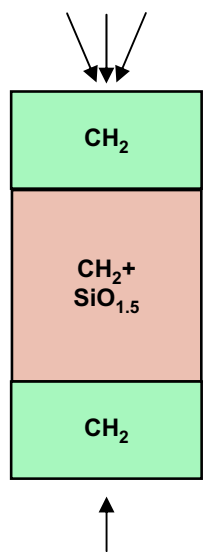

(b)

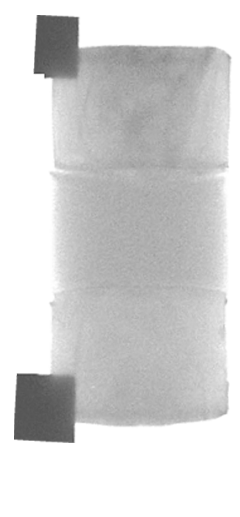

(c)

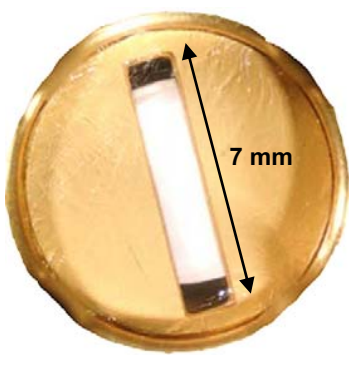

Fig. 6. Target characteristics used in Z1291, an experiment using a restricted field of view and localized dopant. A schematic diagram illustrating the local foam doping over the central 6-mm-tall region is shown in (a). A radiograph of the target recorded before the experiment is shown in (b). An optical photograph of the top of the target after it was attached to the diagnostic aperture is shown in (c). The white foam is visible through the $1.5 \times 7 \mathrm{~mm}$ slot cut into the gold-coated aperture plate. The 7-mm-long slot enables viewing the target beyond the initial 6-mm-diameter. understand the radiation flow into the central region of the hohlraum ahead of the shock.

The other major difference in this second set of experiments was the implementation of a multi-casting fabrication technique that allowed the $\mathrm{SiO}_{1.5}$ dopant to be restricted to the central 6-mm-tall region of the foam. This avoids gradients due to foam plasma that jets out the diagnostic aperture during the experiment. It also provides the basis for future experiments that alter the line opacity by changing the height of the doped region. The disadvantage of these two changes is that the signal is reduced.

Time-resolved spectra recorded in an experiment with the restricted view, as shown in Fig. 6, and the localized dopant are shown in Fig. 7. This experiment (Z1291) used a foam doped over the central 6-mm-region and a $1.5 \times 7 \mathrm{~mm}$ rectangular diagnostic aperture. Thus, the radial profile of the emission across the shock was well-resolved for shock radii of about $1 \mathrm{~mm}$ or greater. Note that the use of a 7-mm-long aperture enabled the observation of emission from any plasma ablated beyond the original foam location by the early time $z$-pinch radiation. Good quality spectra were recorded in the first four frames in this experiment. Schematic diagrams indicating the approximate shock size and the field of view restriction provided by the diagnostic aperture are shown to the right of each frame in Fig. 7. A sequence of lineouts through the images shown in Fig. 7 is shown in Fig. 8. These spectra demonstrated that sufficient signal could be obtained with $\sim 1 \% \mathrm{Si}$ dopant, a 6-mm-tall doped region, and the restricted signal provided when a rectangular aperture was used. In addition, employing a local-dopant in the central 6-mm-tall region greatly reduced the self-absorption of the He-like features, in comparison with the uniform dopant experiment. There is still a residual self-absorbed profile in lines observed on time integrated spectrometers in the vicinity of the cylinder axis. The origin of this residual self-absorption is presently unknown, but may be related to temperature gradients that could exist at the boundary between the doped and undoped region. Some further improvements are clearly possible. The alignment of the images onto the frames was not perfect, so that the emission from the top segment of the shock was missing 

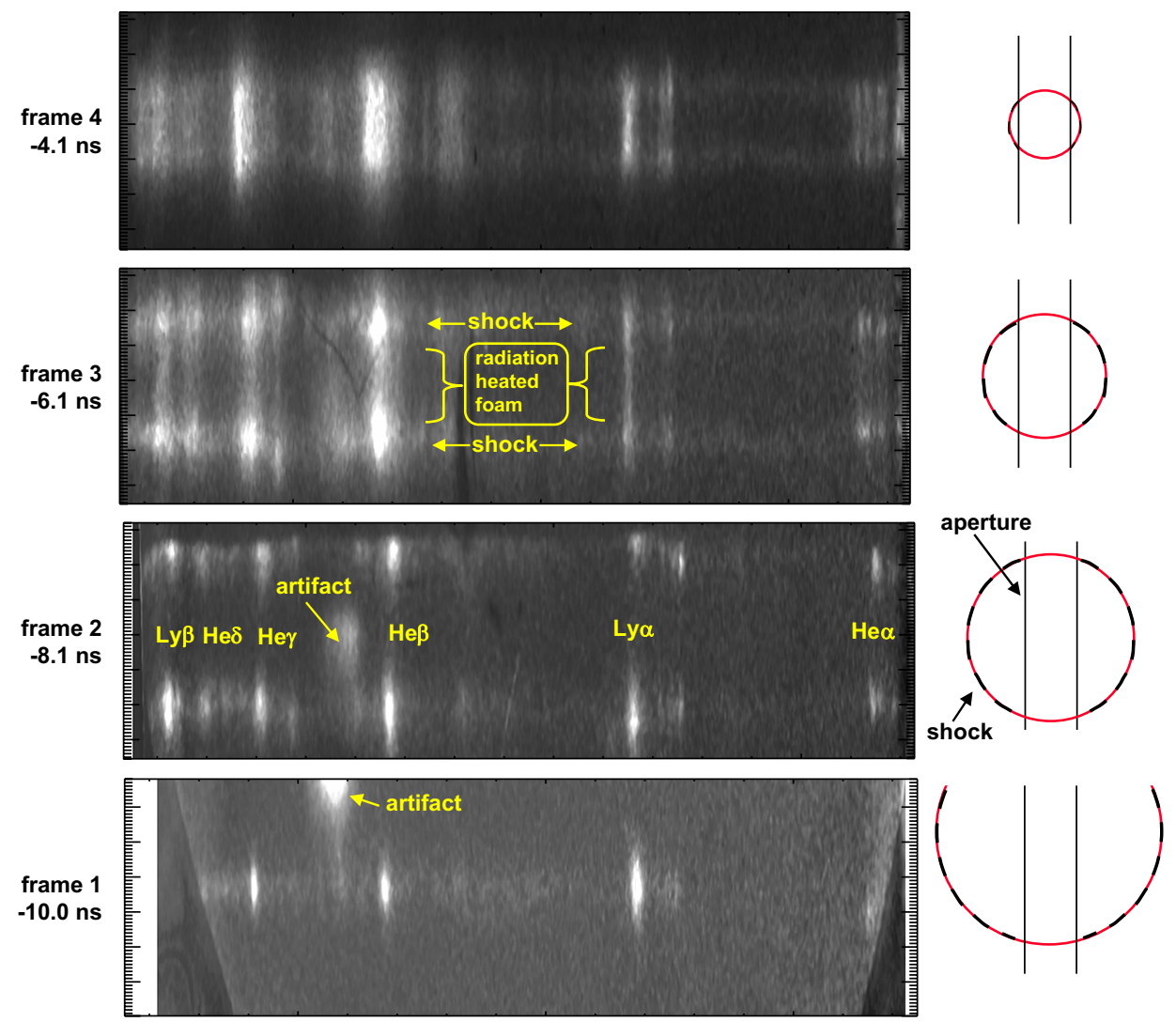

Fig. 7. The space- and time-resolved spectra recorded in the restricted view local-dopant experiment Z1291. The intensity of each image has been adjusted to optimize the display. The approximate shock location and the field of view restriction provided by the rectangular aperture are shown to the side of each image. The shock emission is represented by the red circle and the portion of the shock emission that is blocked by the aperture is marked with a dashed circle. The spaceresolving slit is perpendicular to the long dimension of the slot aperture. The space direction is vertical in each frame and the wavelength axis is horizontal. In frames 1 and 2 the emission arises only from the shocked foam region. In frame 3 the emission from the radiation-heated foam ahead of the shock is clearly visible. In frame 4 the resonance line emission intensity from ahead of the shock is comparable to the intensity from behind the shock.

on frame 1 and partially missing on frame 2 . There are also some artifacts due to MCP and crystal defects. In addition, the electrical gating of the MCP strip lines in this experiment used a $\sim 300$ ps FWHM pulse injected at one end. The opposite end of the strip line was inadvertently operated in an open circuit configuration, so that the pulse reflects. The reflected pulse adds to the incident pulse, but the relative time changes as a function of distance along the strip. The pulse width is comparable to the transit time, so that the applied gate voltage varies with position. Thus, the gain varies by more than an order of magnitude (see Ref. [14]). The pulse was injected at the long wavelength end and consequently the line intensities closer to the long wavelength end are reduced. This makes comparisons of lines separated by more than $0.1-0.2 \AA$ problematic, although comparisons of closely spaced lines, the line profiles, and the spatial dependence of the lines remain valid.

\section{Analysis and discussion}

The focus of our analysis efforts is on the experiment shown in Fig. 7, as it provides almost-pure radial resolution and the local doping avoids the complication associated with absorption by cooler plasma in the vicinity of the diagnostic aperture. The frame 2 and frame 3 spectral images in Fig. 7 consist of bright emission features at radii of approximately $2.2 \mathrm{~mm}$ and $1.66 \mathrm{~mm}$, respectively. In frame 4 the free-free continuum and optically thin satellites to the Ly $\alpha$ have welldefined bright emission features that peak at approximately $0.93 \mathrm{~mm}$ radius, while the resonance line intensities are fairly constant with radius. We associate the bright emission feature in these three frames with the shock, since the radius as a function of time determined from the spectra is essentially the same as the shock radius measured with a filtered time-resolved pinhole camera. The feature in frame 1 is also presumed to be associated with the shock, although the instrument misalignment precludes the determination of the radius. The shock velocity inferred from the spectral data is $\sim 350 \mu \mathrm{m} / \mathrm{ns}$, which is in good agreement with pinhole camera data. Assuming constant velocity, the radius in frame 1 is approximately $2.9 \mathrm{~mm}$, comparable to the initial foam radius. The emission feature spatial width is approximately $1 \mathrm{~mm}$ in frames $1-3$, significantly larger than the $\sim 250 \mu \mathrm{m}$ width measured with pinhole imaging in previous experiments using undoped foam. This difference is not understood, but it could be due in part to defects in the construction or alignment of the foam cylinder with respect to the $z$-pinch. 

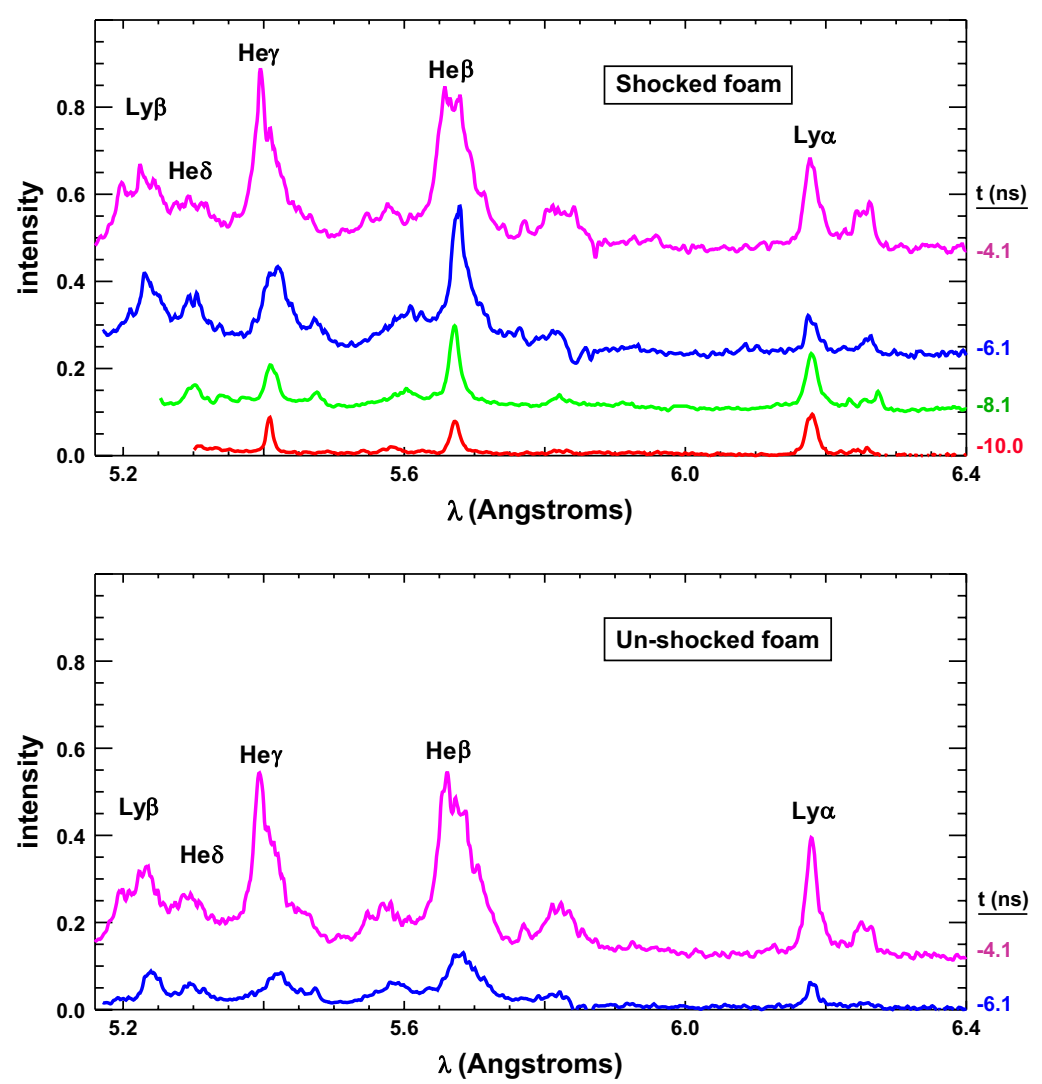

Fig. 8. Lineouts from the shocked and unshocked foam regions in experiment Z1291. The intensity scales are the same for each lineout in order to illustrate the intensity variation with time and space, but each lineout is displaced vertically to enable a clear display. Lineouts from the unshocked region are not shown for frames 1 and 2, since no spectral lines are visible at these times. Note that these lineouts are averages over the indicated regions, but interesting spatial variations are present in the vicinity of the shock.

In the frames 1 and 2 data, no emission is observed from the central region of the foam, ahead of the shock. This does not rule out the possibility that a relatively narrow $(\sim$ few hundred micron) region ahead of the shock front is photopumped by sufficiently strong emission from behind the shock to contribute some of the observed emission on the inside edge of the shock feature. Later in time (frames 3 and 4) emission from the hohlraum interior ahead of the shock becomes bright enough to be measured. Indeed, the spatial profile of the optically thick Ly $\alpha, \mathrm{He} \beta$ and $\mathrm{He} \gamma$ lines is fairly constant with radius in frame 4 . The important qualitative observation from this experimental configuration is that emission lines which should be sensitive to the plasma conditions are recorded from both the shocked foam and from the radiation-heated foam ahead of the shock, at times of interest for ICF capsule experiments.

Although we will not endeavor here to infer quantitative information from the experimental data, we can, nevertheless, gain insight by examination of variations in calculated synthetic spectra. A sequence of calculations at different plasma temperatures and densities was performed with the PRISMSPECT code [15]. This code calculates detailed NLTE atomic populations, including the relevant collisional processes. An optional radiation field can also be included, as photoionization, photoexcitation, and opacity can be included self-consistently in the calculation of the populations. Emergent spectra are calculated for a specified plasma thickness, including opacity effects and Stark broadening [16]. The spectral calculations include the combined effects of $\mathrm{Si}, \mathrm{O}, \mathrm{C}$, and $\mathrm{H}$ atoms present in the experiment of interest. However, the Stark broadening calculations do not include the ion dynamic effects on the $\mathrm{Si}$ emission due to the presence of $\mathrm{H}$ and $\mathrm{C}$ atoms, which will not affect the qualitative nature of the discussion to follow. The other major approximation in these calculations is that, by definition, the calculated emergent spectra represent the emission from a uniform plasma at a single temperature and density. The effects of the local radiation field are calculated self-consistently, but the possible photo processes due to emission at other locations in the plasma are not included. For example, strong Si line emission from the shocked plasma can potentially pump the levels in the unshocked plasma (see below). Work is in progress to incorporate such non-local processes in the SPECT3D code. Despite these approximations, single point calculations can be quickly run for many plasma conditions, providing insight into the trends expected for the spectra.

An example of a set of PRISMSPECT calculations is shown in Fig. 9. These NLTE calculations were all run with an externally imposed Planckian radiation field with $T_{\mathrm{r}}=187 \mathrm{eV}$. The ordinate for the left hand side spectra is 

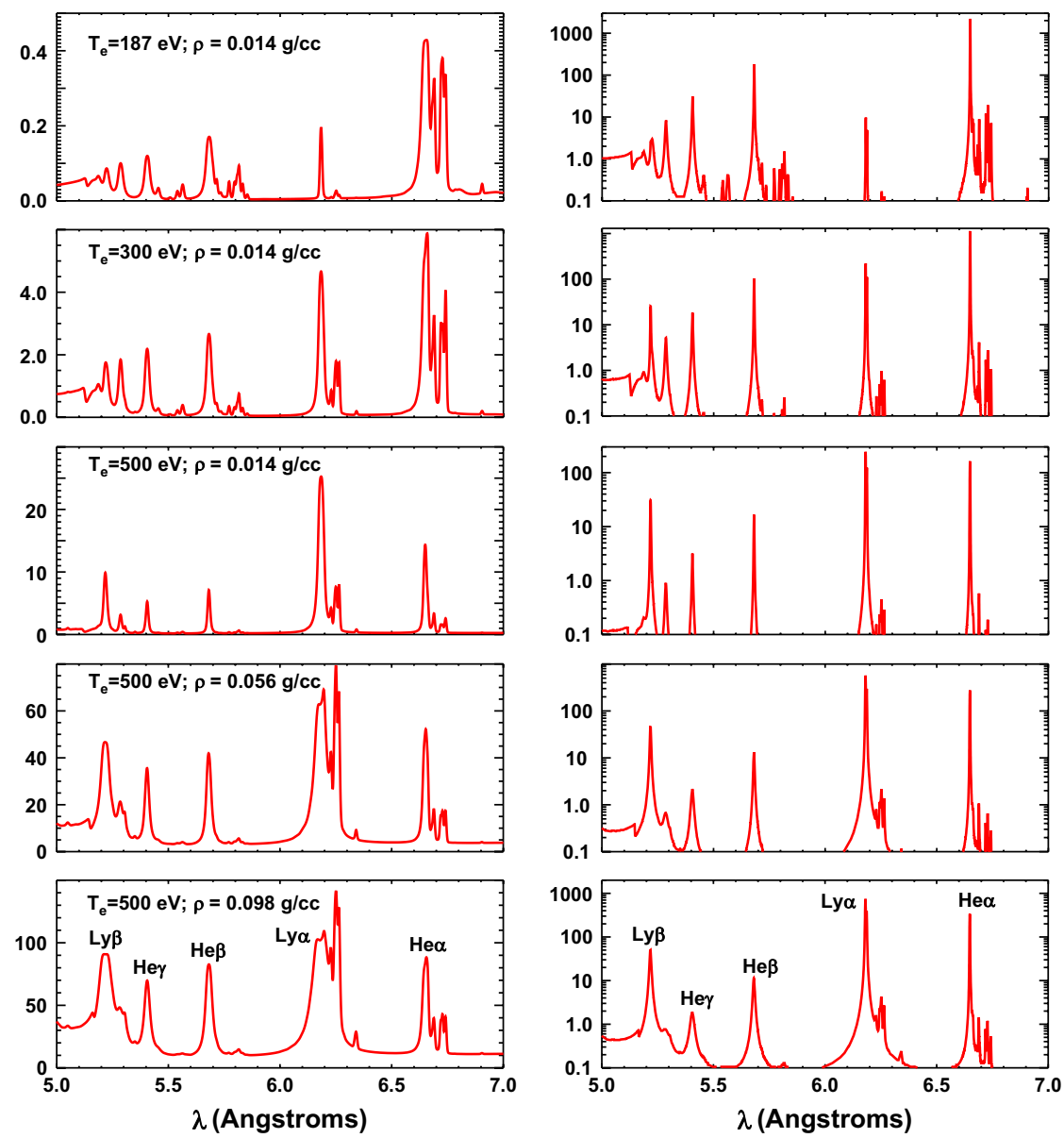

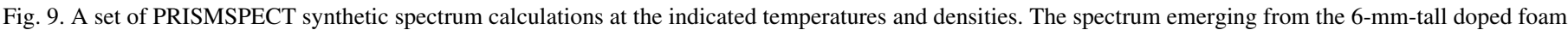
is shown on the left and the corresponding optical depth is shown on the right.

the emergent intensity calculated for a 6-mm-tall slab of plasma at the specified density and temperature. The ordinate for the right hand plots is the optical depth at the same conditions. The spectra have the instrumental resolution included by convolution. These calculations indicate that the absolute emergent intensity increases dramatically with increasing temperature and density. This may be an extremely valuable dependence, even though the present instrument is not absolutely calibrated. Changes in the conditions as a function of time or space should produce easily measurable changes in the intensity. Other potentially useful characteristics of these spectra include the variation in relative line intensities from different charge states as a function of temperature and the growth in the line widths with increasing density. Note that the optical depth plots indicate that the resonance lines are substantially optically thick for the conditions calculated here. Only some of the satellites and possibly the He $\delta$ line are optically thin. This implies that an accurate accounting of opacity is crucial to the analysis. Comparisons with optically thin calculations show that in most cases, optical depth effects account for most of the observed line width.

The top spectrum in Fig. 9 at $T=187 \mathrm{eV}$ and $r=0.014 \mathrm{~g} /$ cc is comparable to conditions predicted ahead of the shock (see Figs. 2 and 3). The spectra at higher temperature and density values are closer to expected characteristics of the shocked foam. Armed with these observations, the simulation results described above imply that the emission from ahead of the shock should be 10-100 times weaker than behind the shock. This expectation is not matched by the experiment, after $t \sim-6 \mathrm{~ns}$. Thus, either the simulation results are not very similar to the experiment, or one of the approximations employed in the PRISMSPECT calculations is not valid. The results of more complete calculations using the WIS model (see below) show that neglecting the non-local photopumping processes is responsible for a major portion of this discrepancy. These calculations imply that $\mathrm{Si}$ line emission from the shocked foam region plays a major role in exciting the line emission from the region ahead of the shock. The simulation fidelity is still an open question that must be tested.

For comparison with the experiments it is important to remember that the data in Fig. 7 are not suitable for comparing line intensities unless the lines are very close together and that the spatial direction for the spectra in Fig. 4 is a blend of radial locations (caused by the circular aperture). However, the spectra in Fig. 7, which are from the experiment with the restricted view and the localized $\mathrm{SiO}_{1.5}$ (schematically illustrated in Fig. 6) demonstrate that for shock radii of less than $\sim 1 \mathrm{~mm}$ the spectral emission ahead of the shock is comparable to the emission from behind the shock. Then the relative intensities from the data in Fig. 4 can be considered qualitatively 


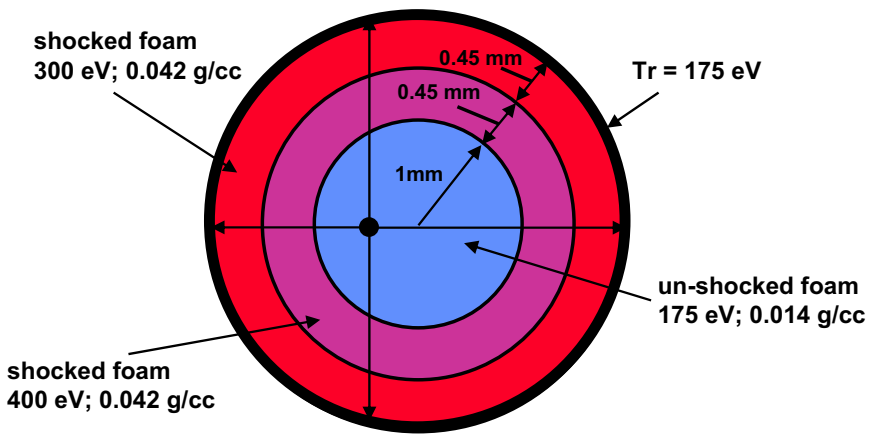

Fig. 10. Schematic diagram of the WIS model calculations used to evaluate the importance on non-local radiation on the spectra emerging from the unshocked foam. The radiation transport equation is solved along the four directions indicated plus the two directions in and out of the page, for a single location within each zone.

characteristic of either the shocked or unshocked foam. Note that this is a broad observation and there are in fact some potentially important differences between the shocked and unshocked foam emissions.

A different analysis approach was pursued using a collisional-radiative model constructed at the Weizmann Institute of Science (the WIS model [17]). This model also employs an NLTE calculation of the level populations accounting for collisional and radiative processes. This model was tailored specifically to this problem, including as intrinsic line shapes detailed calculations of the Stark broadening that account for the $\mathrm{H}$ and $\mathrm{C}$ ion dynamics effect on the Si line profile [18]. In addition, this model uses a two dimensional treatment of the radiation field that enables the non-local photopumping to be included. For this purpose the plasma is divided into a three-zone system as shown in Fig. 10. The central zone represents the unshocked foam and the shocked region is divided into two separate regions. For each zone the radiation transport equation is solved self-consistently with the population kinetics. Computational limitations restrict this solution to one location per zone, selected to be the position half way between the center of the zone and the edge. The transport equation is solved for a set of six ray paths along each of the orthogonal coordinate directions. This provides a preliminary approximation for the total radiation field at that location, including the Planckian radiation that fills the hohlraum, the line and continuum radiation originating from elsewhere within the plasma, and the line and continuum radiation originating from the local region. The total radiation determined for the single location within each zone is then used as an approximation for the radiation field everywhere within the zone. This enables the calculation of emergent spectra as a function of radius for a specified set of plasma conditions within the three zones. These calculations are intensive and the number of excited levels was reduced as a compromise to speed up the computations. More detailed kinetics can be used as the analysis is refined.

The WIS model was used to address the question of whether non-local radiative processes have an important effect on the populations, particularly for the unshocked foam. The unshocked foam properties are the most likely to be influenced by non-local radiation, since the temperature is expected to be lower. Calculations were performed using conditions suggested by the simulations (see Fig. 10). Temperatures of 300-400 eV were chosen for the shocked region, since higher temperatures will make any non-local photopumping effects even more important. The calculated spectrum emerging from the top of the unshocked foam is shown as a red curve in Fig. 11. To address the question of non-local photopumping importance, a second set of calculations performed with the line radiation emitted by the shocked foam switched off is shown as the blue curve in Fig. 11. This calculation demonstrates that the excited states in the unshocked foam are mainly populated by photopumping, since the emission is orders of magnitude smaller when the photopumping is not included.

The conclusion that non-local photopumping is responsible for the line emission emerging from the unshocked foam is consistent with the data shown in Fig. 7. In particular, the similarity of the spectra from ahead and behind the shock in frame 4 would be difficult to explain if this effect was not included. Nevertheless, the model is rather complex
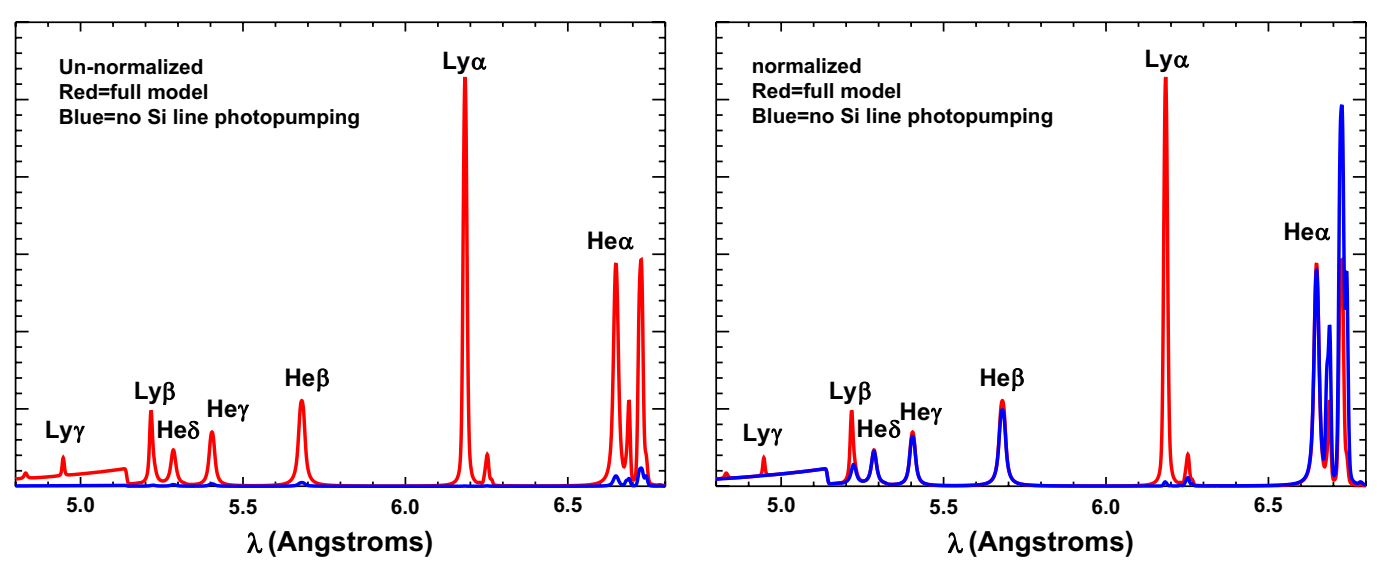

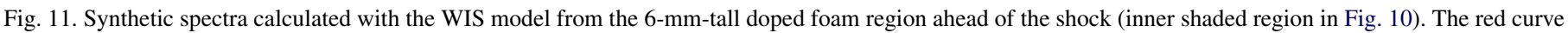
corresponds to the full radiation field calculation and the blue curve has the line emission from the two shocked regions turned off. 
and further scrutiny is in progress. Assuming that this conclusion is substantiated, an analysis strategy can now be formulated. We intend to analyze the plasma conditions from the shocked foam first, since non-local radiation effects are either small or negligible for this region. This allows the plasma conditions to be inferred from the locally observed line intensities and profiles. Once the shocked foam characteristics are determined, synthetic spectra emerging from the unshocked foam, accounting for the non-local radiation, can be computed for a variety of unshocked foam conditions. Comparison of these synthetic spectra with data should lead to a determination of the conditions in the unshocked foam. In the unshocked foam the dominant heating mechanism is still the Planckian radiation field filling the hohlraum and the dominant ionization mechanism is collisional ionization by the free electrons. Therefore, the emergent spectra are still expected to be sensitive to the local temperature and density conditions, even though the predominant excitation is due to the non-local radiation. The interpretation of such data in the presence of combined thermal and non-thermal radiation fields is complex. However, we regard the effective photopumping processes to be beneficial, since otherwise it might be very difficult to observe emission spectra from the radiation-heated plasma ahead of the shock.

The successful interpretation of these data should lead to greatly improved understanding of the radiation available for driving ICF capsule implosions. In addition, these plasmas have intrinsic interest, as the ability to create a uniform 6$\mathrm{mm}$-scale plasma heated by radiation to greater than $200 \mathrm{eV}$ is unique to the best of our knowledge. It is conceivable that these plasmas may be useful for evaluation of Stark broadening calculations, since the initial density is known. This depends on whether an early ablation shock significantly alters the initial conditions. Stark broadening calibrations would obviously require lower dopant levels to reduce opacity broadening, but this should be easily achievable. Alternatively, such plasmas may be useful for opacity studies. A major challenge for this application is the development of a backlighter source bright enough to overwhelm the self-emission. The $\mathrm{Z}$ beamlet laser may be suitable for this purpose. The possibility of applying $\mathrm{dy}-$ namic hohlraum plasmas for these applications will be clarified by quantitative interpretation of the actual plasma conditions.

\section{Acknowledgements}

We thank the $\mathrm{Z}$ accelerator, diagnostics, materials processing, target fabrication, and wire array teams for invaluable and dedicated technical assistance. L.P. Mix provided data processing assistance and R.C. Mancini provided comments on the manuscript. We are also grateful to R.J. Leeper and M.K. Matzen for support and encouragement. Sandia is a multiprogram laboratory operated by Sandia Corporation, a Lockheed Martin Company, for the U.S. Department of Energy under contract No. DE-AC04-94AL85000.

\section{References}

[1] M.K. Matzen, Phys. Plasmas 4 (1997) 1519.

[2] V.P. Smirnov, Plasma Phys. Controlled Fusion 33 (1991) 1697.

[3] J.H. Brownell, R.L. Bowers, K.D. McLenthan, D.L. Peterson, Phys. Plasmas 5 (1998) 2071.

[4] S.A. Slutz, J.E. Bailey, G.A. Chandler, G.R. Bennett, G. Cooper, J.S. Lash, et al., Phys. Plasmas 10 (2003) 1875.

[5] J.E. Bailey, G.A. Chandler, S.A. Slutz, I. Golovkin, P.W. Lake, J.J. MacFarlane, et al., Phys. Rev. Lett. 92 (2004) 085002.

[6] C.L. Ruiz, G.W. Cooper, S.A. Slutz, J.E. Bailey, G.A. Chandler, T.J. Nash, et al., Phys. Rev. Lett. 93 (2004) 015001.

[7] R.W. Lemke, J.E. Bailey, G.A. Chandler, T.J. Nash, S.A. Slutz, T.A. Mehlhorn, et al., Phys. Plasmas 12 (2004) 012703.

[8] G. Zimmerman, W. Kruer, Comments Plasma Phys. Controlled Fusion 2 (1975) 85

[9] J.E. Bailey, G.A. Chandler, S.A. Slutz, G.R. Bennett, G. Cooper, J.S. Lash, et al., Phys. Rev. Lett. 89 (2002) 095004.

[10] S.A. Slutz, in preparation.

[11] B.L. Henke, H.T. Yamada, T.J. Tanaka, Rev. Sci. Instrum. 54 (1983) 1311.

[12] P.W. Lake, J.E. Bailey, G.A. Rochau, T.C. Moore, D. Petmecky, P. Gard, Rev. Sci. Instrum. 75 (2004) 3690.

[13] T.J. Nash, M.S. Derzon, G.A. Chandler, D.L. Fehl, R.J. Leeper, J.L. Porter, et al., Rev. Sci. Instrum. 72 (2001) 1167

[14] J.D. Kilkenny, Laser Part. Beams 9 (1991) 49-69.

[15] J.J. MacFarlane, I.E. Golovkin, P.R. Woodruff, in: B. Hammel, D. Meyerhofer, J. Meyerter Vehn, H. Azechi (Eds.), Proceedings of inertial fusion science and applications 2003, American Nuclear Society, La Grange Park, Il, 2004, p. 457.

[16] D.A. Haynes, Private communication, 2003.

[17] V. Fisher, Y. Maron, Private communication, 2005.

[18] E. Stambulchik, Y. Maron, I. J. Almiev, J. Quant. Spectrosc. Radiat. Transf. this issue. E. Stambulchik, K. Tsigutkin, Y. Maron, Europhysics Conference Abstracts 27A (2003) 1.58. 